\title{
Analysis of expressed sequence tags generated from full-length enriched cDNA libraries of melon
}

\author{
Christian Clepet ${ }^{1}$, Tarek Joobeur ${ }^{2,10}$, Yi Zheng $^{3}$, Delphine Jublot ${ }^{1}$, Mingyun Huang ${ }^{3}$, Veronica Truniger ${ }^{4}$, \\ Adnane Boualem¹, Maria Elena Hernandez-Gonzalez², Ramon Dolcet-Sanjuann, Vitaly Portnoy ${ }^{6}$, \\ Albert Mascarell-Creus ${ }^{7}$, Ana I Caño-Delgado ${ }^{7}$, Nurit Katzir ${ }^{6}$, Abdelhafid Bendahmane ${ }^{1,8}$, James J Giovannoni ${ }^{3,9}$, \\ Miguel A Aranda ${ }^{4}$, Jordi Garcia-Mas ${ }^{5}$ and Zhangjun Fei $i^{3,9^{*}}$
}

\begin{abstract}
Background: Melon (Cucumis melo), an economically important vegetable crop, belongs to the Cucurbitaceae family which includes several other important crops such as watermelon, cucumber, and pumpkin. It has served as a model system for sex determination and vascular biology studies. However, genomic resources currently available for melon are limited.

Result: We constructed eleven full-length enriched and four standard cDNA libraries from fruits, flowers, leaves, roots, cotyledons, and calluses of four different melon genotypes, and generated 71,577 and 22,179 ESTs from fulllength enriched and standard cDNA libraries, respectively. These ESTs, together with 35,000 ESTs available in public domains, were assembled into 24,444 unigenes, which were extensively annotated by comparing their sequences to different protein and functional domain databases, assigning them Gene Ontology (GO) terms, and mapping them onto metabolic pathways. Comparative analysis of melon unigenes and other plant genomes revealed that $75 \%$ to $85 \%$ of melon unigenes had homologs in other dicot plants, while approximately $70 \%$ had homologs in monocot plants. The analysis also identified 6,972 gene families that were conserved across dicot and monocot plants, and 181, 1,192, and 220 gene families specific to fleshy fruit-bearing plants, the Cucurbitaceae family, and melon, respectively. Digital expression analysis identified a total of 175 tissue-specific genes, which provides a valuable gene sequence resource for future genomics and functional studies. Furthermore, we identified 4,068 simple sequence repeats (SSRs) and 3,073 single nucleotide polymorphisms (SNPs) in the melon EST collection. Finally, we obtained a total of 1,382 melon full-length transcripts through the analysis of full-length enriched cDNA clones that were sequenced from both ends. Analysis of these full-length transcripts indicated that sizes of melon $5^{\prime}$ and $3^{\prime}$ UTRs were similar to those of tomato, but longer than many other dicot plants. Codon usages of melon full-length transcripts were largely similar to those of Arabidopsis coding sequences.

Conclusion: The collection of melon ESTs generated from full-length enriched and standard cDNA libraries is expected to play significant roles in annotating the melon genome. The ESTs and associated analysis results will be useful resources for gene discovery, functional analysis, marker-assisted breeding of melon and closely related species, comparative genomic studies and for gaining insights into gene expression patterns.
\end{abstract}

\footnotetext{
* Correspondence: zf25@cornell.edu

${ }^{3}$ Boyce Thompson Institute, Cornell University, Ithaca, NY 14853, USA

Full list of author information is available at the end of the article
} 


\section{Background}

Melon (Cucumis melo) belongs to the Cucurbitaceae family, which comprises 130 genera, including approximately 800 species that are mainly found in temperate, subtropical and tropical regions worldwide [1,2]. Besides melon, the Cucurbitaceae family also consists of many other economically important species, including cucumber (C. sativus), watermelon (Citrullus lanatus), squash and pumpkin (Cucurbita spp.). Economically, melon is among the most important fleshy fruits for fresh consumption. Indeed, melon is one of America's, Europe's and the Middle East's favorite fruits for dessert and salad uses because of its unique flavor. The average per capita consumption of melon in the U.S. has been increasing consecutively each decade since the 1960s with 2000-2006 average per capita consumption exceeding 12 pounds per year, an 8\% rise from 1990-1999. Besides its economic importance, melon is a very useful experimental system for fundamental studies on a range of topics including sex determination [3,4] and vascular biology $[5,6]$. In addition, melon is also an intensively studied species in terms of fruit ripening. It exhibits extreme diversity for fruit traits and includes a wide variety of cultivars producing fruits differing in many traits including fruit shape, size, flesh color, sweetness, aroma volatiles and fruit texture [7]. In addition, melon fruits also have significant variations in ripening physiology and can be categorized as either climacteric or nonclimacteric types based on their ripening related respiration rate and ethylene evolution profiles [8]. Extensive molecular and genetic studies have been carried out in recent years in order to better understand the regulatory mechanisms underlying important traits of melon with the aim to improve melon fruit quality $[9,10]$

Melon is a diploid species $(2 \mathrm{n}=24)$ with an estimated genome size of $450 \mathrm{Mb}$ [11]. Genetic and genomic tools available in melon include BAC libraries [12-14], a physical map [15], high-resolution genetic maps [16-19], oligo-based microarrays [20], and a TILLING platform for functional studies [21]. Currently the melon genome is being sequenced under the Spanish Genomics Initiative (MELONOMICS) and the genome sequencing should be completed in the near future. The sequence of the closely related cucumber genome is available [22]. Complementary to whole genome sequences, expressed sequence tags (ESTs) can directly represent the transcriptome or transcribed portions of the genome. They have played significant roles in rapid gene discovery, improving genome annotation, elucidating phylogenetic relationships, facilitating breeding programs, and largescale expression analysis [23]. Currently in the NCBI dbEST database, there are approximately 35,000 melon ESTs, most of which were produced by González-Ibéas et al. [24]. Approximately 8,000 ESTs are available for cucumber and watermelon, respectively, and a total of approximately 1,000 EST from other cucurbit species. Recently several reports have described the generation of large-scale transcriptome sequences in cucurbit species using next generation sequencing technologies (mainly the Roche-454 massive parallel pyrosequencing technology), including melon [25], cucumber [26], and Cucurbita pepo [27]. Although sequences generated under these efforts are much shorter than traditional Sanger ESTs, they represent a significant expansion of cucurbit functional genomics resources.

We undertook to expand the melon transcript catalog in the framework of the International Cucurbit Genome Initiative, which was established in 2005 , being one of its major objectives to sequence approximately 100,000 ESTs from different melon genotypes and tissues [28]. We have constructed eleven full-length enriched cDNA libraries and four standard cDNA libraries from various melon tissues and cultivars and generated $\sim 94,000$ ESTs. These melon ESTs were analyzed to determine the structure and putative functions of the corresponding transcripts. In addition, a number of new SSR and SNP markers were identified in this EST collection. All of this data has been integrated in the Cucurbit Genomics Database [28]. The ESTs generated from the present study, especially those from full-length enriched cDNA libraries, will be a useful resource for the ongoing melon whole genome sequencing project and for characterizing gene expression patterns and traits of interest in melon and closely related species.

\section{Results and discussion}

\section{Construction and sequencing of melon cDNA libraries}

We constructed eleven full-length enriched and four standard cDNA libraries from various melon tissues (cotyledon, leaf, root, flower, fruit and callus) and cultivars (Dulce, PI161375, Piel de Sapo T-111, and Vedrantais) under normal conditions or upon infection with melon necrotic spot virus (MNSV)-M $\alpha 5$ (Table 1). The flower, fruit and callus libraries were derived from two climacteric (Dulce and Vedrantais) and two non-climacteric cultivars (Piel de sapo T-111 and PI161375). For the flower and fruit, RNA pools were prepared from various developmental stages (see Methods). The leaf, root and cotyledon libraries were constructed from tissues infected with MNSV-M $\alpha 5$. EST sequencing was carried out independently on full-length enriched and standard cDNA clones. For full-length enriched cDNA libraries, 70,576 randomly-selected clones were sequenced from the 5' end, producing 69,196 (98\%) useful reads after trimming vector, adaptor and low-quality sequences and identifying and removing all possible contaminated sequences. Assembly of these ESTs produced 6,469 clusters, among which 2,721 non-redundant 
Table 1 Description of melon cDNA libraries and summary of melon ESTs

\begin{tabular}{|c|c|c|c|c|c|}
\hline Library & Cultivar & Tissue & No. $5^{\prime}$ sequences & No. 3' sequences & Total sequences \\
\hline \multicolumn{6}{|c|}{ Full-length cDNA library } \\
\hline PFTP2 & PI161375 & mixture of fruits in four developmental stages & 12,673 & 547 & 13,220 \\
\hline SFTP2 & Piel de Sapo T-111 & mixture of fruits in four developmental stages & 3,630 & 139 & 3,769 \\
\hline VFTP2 & Vedrantais & mixture of fruits in four developmental stages & 3,621 & 149 & 3,770 \\
\hline DFTP2 & Dulce & mixture of fruits in four developmental stages & 3,549 & 70 & 3,619 \\
\hline VFLP2 & Vedrantais & mixture of flowers in three developmental stages & 19,261 & 638 & 19,899 \\
\hline PFLP2 & PI161375 & mixture of flowers in three developmental stages & 3,548 & 62 & 3,610 \\
\hline DFLP2 & Dulce & mixture of flowers in three developmental stages & 3,411 & 126 & 3,537 \\
\hline SFLP2 & Piel de Sapo T-111 & mixture of flowers in three developmental stages & 320 & 0 & 320 \\
\hline MNFG2 & Piel de Sapo T-111 & leaf infected by melon necrotic spot virus (MNSV) & 7,776 & 295 & 8,071 \\
\hline MNRP2 & Piel de Sapo T-111 & root infected by melon necrotic spot virus (MNSV) & 7,953 & 297 & 8,250 \\
\hline MNCP2 & Piel de Sapo T-111 & cotyledon infected by melon necrotic spot virus (MNSV) & 3,454 & 58 & 3,512 \\
\hline \multicolumn{3}{|c|}{ Subtotal No. sequences } & 69,196 & 2,381 & 71,577 \\
\hline \multicolumn{6}{|c|}{ Standard cDNA libraries } \\
\hline CM-DEa & Dulce & callus & 5,485 & 0 & 5,485 \\
\hline CM-PEa & PI161375 & callus & 5,527 & 0 & 5,527 \\
\hline CM-TEa & Piel de Sapo & callus & 5,700 & 0 & 5,700 \\
\hline CM-VEa & Vedrantais & callus & 5,467 & 0 & 5,467 \\
\hline \multicolumn{3}{|c|}{ Subtotal No. sequences } & 22,179 & 0 & 22,179 \\
\hline \multicolumn{3}{|l|}{ Total } & 91,375 & 2,381 & 93,756 \\
\hline
\end{tabular}

clones were selected for 3' end sequencing, yielding a total of 2,381 (87.5\%) high quality 3' reads. For the four standard callus libraries, 26,112 randomly-selected clones were sequenced from the 5 ' end, generating 22,179 (85\%) high quality EST sequences. In total, we have generated 93,756 high quality melon ESTs from the constructed cDNA libraries (Table 1) and the average length of these ESTs is 629.6 bp. The EST sequences have been deposited in GenBank and are also available at the Cucurbit Genomics Database [28].

\section{Melon EST sequence assembly and annotation}

The 93,756 high quality melon ESTs generated under this study, together with $\sim 35,000$ ESTs that are publicly available $[24,28,29]$ and 173 published mRNA sequences, were assembled into a melon unigene build. The resulting assembly contained a total of 24,444 unigenes with an average length of $776.7 \mathrm{bp}$, among which 11,653 were contigs with an average length of $972 \mathrm{bp}$ and 12,791 were singletons with an average length of 598.7 bp (Table 2). The distribution of the number of ESTs in each melon unigene is shown in Figure 1. A number of highly abundant genes could be identified, with 162

Table 2 Statistics of melon unigenes

\begin{tabular}{llll}
\hline & Singleton & Contig & Unigene \\
\hline No. of sequences & 12,791 & 11,653 & 24,444 \\
Average read length (bp) & 598.7 & 972.0 & 776.7 \\
Total bases (bp) & $7,658,604$ & $11,326,166$ & $18,984,770$ \\
\hline
\end{tabular}

unigenes represented by over 100 ESTs. The most abundant genes in the combined set of libraries (> 500 ESTs) are listed in Table 3. Details of the melon EST assembly are available at the Cucurbit Genomics Database [28].

Putative functions of melon unigenes were accessed by comparing unigene sequences against the GenBank nonredundant $(\mathrm{nr})$ protein database using the NCBI BLAST program. The analysis showed that applying an e value cutoff of 1e-5, a total of 19,359 (79.2\%) melon unigenes had hits in the nr database; while a total of 10,068 (41.2\%) had hits when an e value cutoff of 1 e-50 was applied. This indicated that a very high percentage of melon unigenes could be assigned a putative function. Those having no hits in the database are likely to

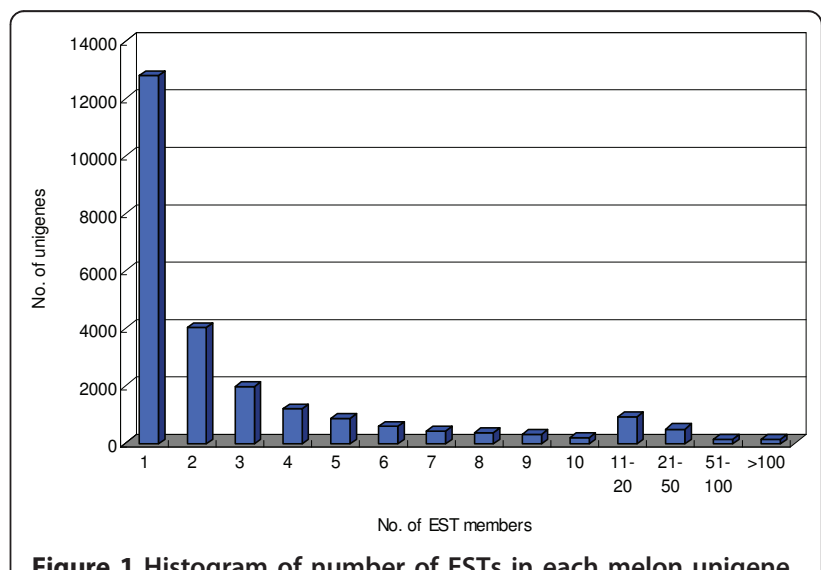


Table 3 Most abundant melon unigenes (>500 EST members)

\begin{tabular}{|c|c|c|c|}
\hline $\begin{array}{l}\text { Unigene } \\
\text { ID }\end{array}$ & $\begin{array}{l}\text { No. of } \\
\text { ESTs }\end{array}$ & GenBank nr hit description & $\begin{array}{l}\mathrm{E} \\
\text { value }\end{array}$ \\
\hline MU46026 & 2054 & $\begin{array}{l}\text { ELP (EXTENSIN-LIKE PROTEIN); lipid } \\
\text { binding }\end{array}$ & $1 e-31$ \\
\hline MU45978 & 1120 & type I proteinase inhibitor-like protein & $9 e-06$ \\
\hline MU46015 & 915 & acyl carrier protein & $1 e-25$ \\
\hline MU45913 & 827 & type-2 metallothionein & $2 \mathrm{e}-19$ \\
\hline MU45877 & 819 & No hits found & \\
\hline MU45416 & 786 & No hits found & \\
\hline MU45994 & 785 & B12D-like protein & $3 e-38$ \\
\hline MU46019 & 683 & Wound-induced proteinase inhibitor 1 & $1 e-10$ \\
\hline MU45854 & 626 & No hits found & \\
\hline MU45964 & 619 & lipid binding protein & $3 e-16$ \\
\hline MU43757 & 618 & 60 s acidic ribosomal protein & $4 e-23$ \\
\hline MU47776 & 594 & histone cluster 2, H3c2-like & $2 \mathrm{e}-60$ \\
\hline MU45763 & 591 & type-2 metallothionein & $2 \mathrm{e}-19$ \\
\hline MU47828 & 580 & $\begin{array}{l}\text { chloroplast photosystem \| } 10 \mathrm{kDa} \\
\text { protein }\end{array}$ & $1 e-52$ \\
\hline MU45654 & 568 & chlorophyll A/B binding protein & $7 e-147$ \\
\hline MU45963 & 554 & histone $\mathrm{H} 4$ & $3 e-38$ \\
\hline MU45282 & 514 & ascorbate peroxidase & $1 e-120$ \\
\hline MU45991 & 509 & ubiquitin carrier-like protein & $4 e-83$ \\
\hline
\end{tabular}

include non-coding RNAs, genes whose sequences do not capture regions that contain conserved functional domains, or protein coding genes that are novel in the database and/or are melon-specific.

We then further compared melon unigenes to the pfam protein domain database [30]. A total of 8,251 (33.8\%) melon unigenes contained at least one pfam domain and a total of 2,206 distinct pfam domains were represented by these 8,251 melon unigenes. A similar analysis on the well-annotated Arabidopsis proteins (TAIR version 10) indicated that 3,272 pfam domains could be represented by the Arabidopsis proteome. This suggested that melon unigenes assembled in the present study captured a large portion (at least $70 \%$ ) of genes in the melon genome. The most highly represented pfam domains in the melon unigene database included PF00069 (protein kinase; 144 unigenes), PF00076 (RNA recognition motif; 138 unigenes), PF07714 (protein tyrosine kinase; 108 unigenes) and PF00097 (Zinc finger, C3HC4 type; 103 unigenes).

Based on BLAST and pfam annotations, melon unigenes were further annotated with Gene Ontology (GO) terms. A total of 15,350 (62.8\%) unigenes were assigned at least one GO term, among which 12,953 (53\%) were assigned at least one GO term in the biological process category, $13,149(53.8 \%)$ in the molecular function category and $12,420(50.8 \%)$ in the cellular component category; while 9,927 (40.6\%) melon unigenes were annotated with GO terms from all the three categories.
Based on the GO annotations, putative gene functions of melon unigenes were classified into high-level plant specific GO slims [31] in each of the three categories. The most abundant GO slims within the biological process, molecular function, and cellular component categories were cellular process, binding, and membrane, respectively. In addition, a large number of melon unigenes appeared to be involved in plant responses to abiotic $(1,534)$ and biotic (844) stimuli, flower development (347), and secondary metabolite process (603), or have transcription factor activities (519).

To gain insights into metabolism-related genes, we further predicted biochemical pathways from the melon unigenes and built a melon metabolic pathway database using the Pathway Tools software [32]. A total of 302 metabolic pathways, as well as 30 superpathways, were predicted from 3,543 enzyme-coding melon unigenes. Most primary and secondary metabolic pathways were well-represented by melon unigenes. The melon metabolic pathway database is freely available at the Cucurbit Genomics Database [28].

\section{Quality assessment of melon full-length enriched cDNAs}

As shown in Table 1, a total of 71,577 ESTs derived from full-length enriched cDNA clones were obtained in the present study. These ESTs were assembled into 6,848 unigenes, among which 6,469 contained 5' sequences of at least one full-length enriched cDNA clone. By blasting sequences of the 6,469 unigenes against GenBank nr, SwissProt/TrEMBL and Arabidopsis (TAIR version 10) protein databases, 5,552 (85.8\%) had significant hits (1e-05). Out of the 5,552 unigenes, 4,668 (84.1\%) hit within five amino acids of the corresponding start sites. This indicated that a large portion of clones from full-length enriched cDNA libraries encoded full-length cDNAs.

We further generated 3' end sequences of more than 2,300 clones (Table 1 ) and ultimately obtained 2,162 clones that were sequenced from both the 5' and 3' ends, among which 1,538 (72.5\%) had 5' and 3' sequences that were assembled into the same unigene. After removing redundancy, a total of 1,382 unigenes that contained 5' and 3' sequences of at least one fulllength enriched cDNA clone were identified as melon full-length transcripts. The majority of the identified full-length transcripts contained overlapping 5' and 3' sequences from the same clone. The length distribution of melon full-length transcripts is shown in Figure 2A. The full-length transcripts ranged from 269 to 2,839 bp and their average size was $1,230 \mathrm{bp}$, which was shorter than previously reported for tomato $(1,418 \mathrm{bp}$; [33]), Arabidopsis (1,445 bp; [34]), and soybean (1,539 bp; [35]), but longer than poplar (1,045 bp; [36]). We then predicted the complete protein-coding sequences (CDS) 


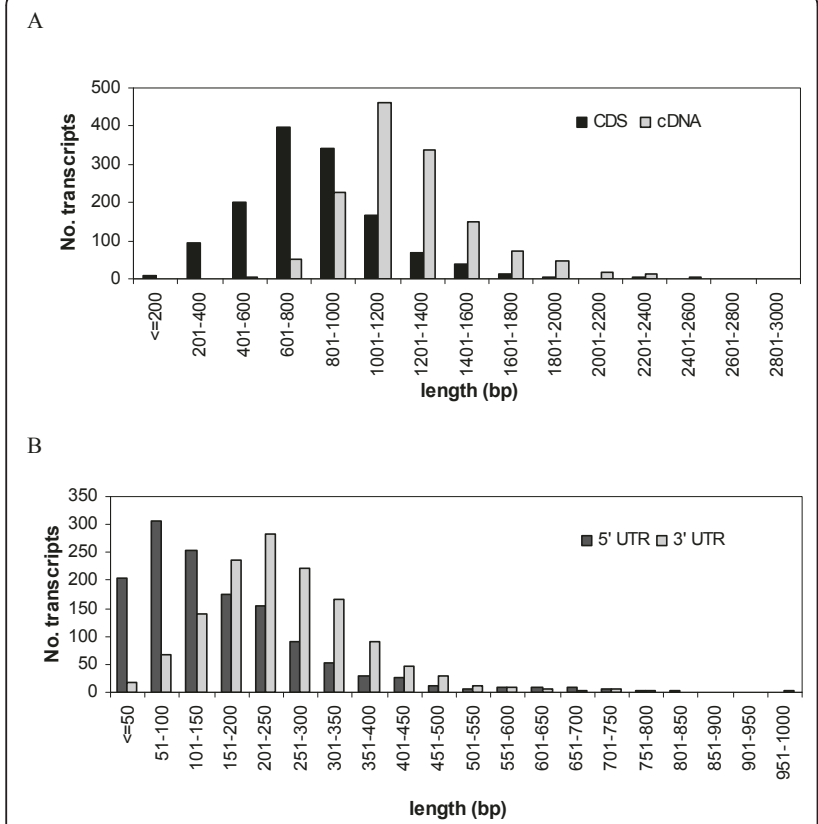

Figure 2 Size distribution of CDNAs, CDS (A) and $5^{\prime}$ and $3^{\prime}$ UTRs (B) of melon full-length transcripts.

for the 1,382 melon full-length transcripts and were able to obtain CDS for 1,345 (97.3\%) full-length transcripts. The remaining 37 could be non-coding RNAs or transcripts that did not contain full CDS. Indeed, we found that four transcripts (e.g., MU51348) did not contain a stop site. The average length of the predicted CDS was $814 \mathrm{bp}$, which was shorter than that of tomato (938 bp; [33]) and soybean (1,042 bp; [35]), but longer than poplar (649 bp; [36]) and maize (799 bp; [37]). The size distribution of melon CDS predicted from melon fulllength transcripts is illustrated in Figure 2A. Overall, the average lengths of both melon full-length transcripts and CDS were shorter than those reported for fulllength cDNAs of other plant species such as tomato [33], Arabidopsis [34], and soybean [35]. This is not unexpected since, as mentioned earlier, the majority of melon full-length transcripts were identified based on the overlap between 5' and 3' sequences of a single fulllength cDNA clone.

Based on the predicted CDS, we extracted 5' and 3' UTR sequences for each melon full-length transcript. The average lengths of melon 5' and 3' UTRs were 167 bp and $254 \mathrm{bp}$, respectively, which were very close to those of tomato (175 bp and $257 \mathrm{bp}$, respectively) and longer than those of other plant species except rice [33]. The length distributions of melon 5' and 3' UTRs are shown in Figure 2B, which were also largely similar to those of tomato [33].

We further examined codon usages of the 1,345 melon full-length transcripts and compared the codon usages to those of Arabidopsis coding sequences (TAIR version 10). The statistics of the complete codon usages of melon and Arabidopsis CDS are provided in Additional file 1. Overall codon usages of melon full-length transcripts were largely similar to those of Arabidopsis CDS. TGA, TAA, and TAG accounted for $44.9 \%, 37.2 \%$, and $17.9 \%$, respectively, of melon stop codons; and they accounted for $43.6 \%, 36 \%$, and $20.4 \%$, respectively, of Arabidopsis stop codons (Additional file 1). In addition, the GC content of melon coding sequences (45.61\%) was also very close to that of Arabidopsis (44.14\%). This, combined with the evidence described above, supported the high quality of melon full-length enriched cDNA libraries.

\section{Comparative genomics analysis with other plants}

To date, genome sequences of fourteen plant species have been published. These plant species are Arabidopsis [38], rice [39], poplar [40], grape [41], papaya [42], sorghum [43], cucumber [22], maize [44], soybean [45], Brachypodium [46], apple [47], castor bean [48], strawberry [49], and cacao [50]. Protein sequences of genes predicted from the fourteen plant genomes were downloaded from corresponding websites (Additional file 2). The 24,444 melon unigenes were then compared to these protein sequence databases using the NCBI BLAST (blastx) program. The complete comparative analysis results are shown in Additional file 3. At e value $<1 \mathrm{e}-05$, approximately $85 \%$ of melon unigenes matched to proteins of cucumber, $75.4 \%$ to $79.2 \%$ of melon unigenes matched proteins of other dicot plants (Arabidopsis, poplar, apple, strawberry, cacao, grape, papaya, soybean, and castor bean), while $70.6 \%$ to $72.5 \%$ of melon unigenes matched proteins of monocot plants (rice, maize, sorghum, and Brachypodium). At a very stringent e value cutoff (e value < 1e-100), approximately $30 \%$ of melon unigenes matched cucumber proteins, $10.8 \%$ to $13.6 \%$ matched proteins of other dicot plants, and $7.9 \%$ to $8.5 \%$ matched proteins of monocot plants (Additional file 3). These matches represented the highly conserved proteins between melon and other plant species.

We constructed families of homologous proteins using OrthoMCL [51] from protein sequences translated from melon unigenes with ESTScan [52] and from a wide phylogenetic range of representative plant organisms including cucumber, Arabidopsis, rice, and grape. These four organisms were chosen for the OrthoMCL analysis because cucumber, as melon, belongs to the Cucurbitaceae family; grape, cucumber and some cultivars of melon (e.g., Piel de sapo) are non-climacteric fleshy fruit; and Arabidopsis and rice represent the model systems for dicot and monocot plants, respectively. As shown in Figure 3, the analysis revealed 6,972 gene families that were distributed among the five genomes, 


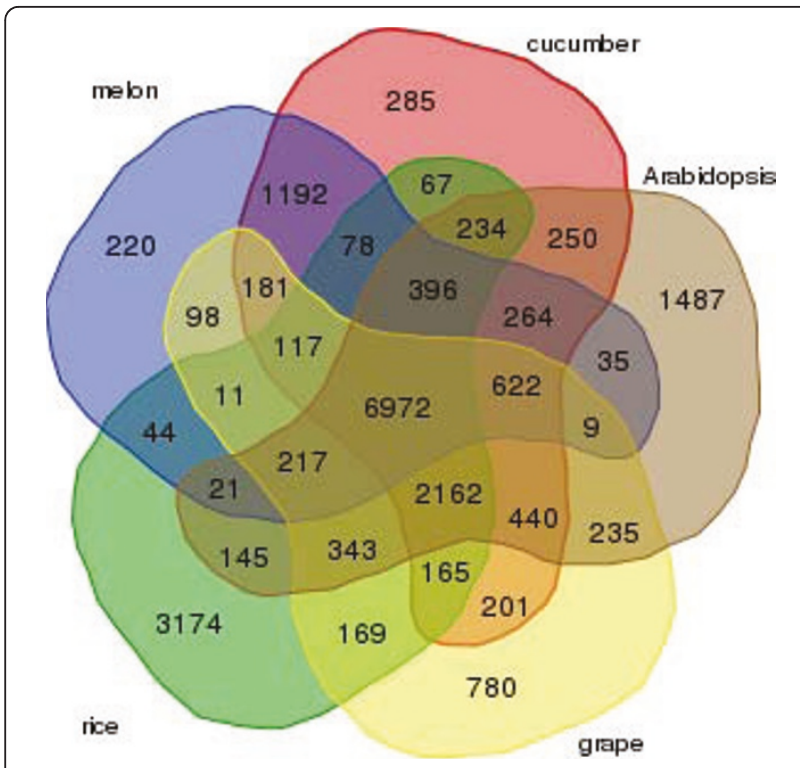

Figure 3 Venn diagram of ortholog group distribution in melon, cucumber, Arabidopsis, grape, and rice. Numbers in individual sections indicate the numbers of ortholog groups which represented highly conserved gene families across dicot and monocot plant kingdoms. We also identified 181 gene families that were specific to fleshy fruit-bearing plants (melon, cucumber, and grape), 1,192 families specific to the Cucurbitaceae family (melon and cucumber), and 220 specific to melon. Functional analysis of melon unigenes using GO terms revealed that the 6,972 melon gene families common to the other four plant species were highly enriched with GO terms related to cellular process, metabolic process, and biosynthetic process (Additional file 4). This is consistent with a previous report [50]. Gene families specific to fleshy fruits were significantly enriched with GO terms related to hormone-mediated signaling pathway, response to biotic stimulus, and regulation of metabolic processes (Additional file 4); all these biological processes have been reported to be related to fleshy fruit development [53]. Gene families specific to the Cucurbitaceae family were significantly enriched with GO terms related to responses to various stimuli including responses to hormone and chemical stimuli (Additional file 4). Both melon and cucumber have diverse floral sex types and have long served as the primary model systems for sex determination studies [54]. It has been reported that a number of environment variables, such as light, temperature, water stress, and disease, as well as exogenous treatment with hormones or other growth-regulating substances, can directly influence floral sex determination $[55,56]$. Results obtained from the OrthoMCL analysis indicated that cucurbit specific gene families were enriched with such stimulus-responsive genes which might play roles in floral sex determination. Further studies, of course, are required to test this hypothesis. Finally, we found that gene families specific to melon mainly encompassed genes of unknown functions, which is consistent with findings reported in other plant species [50].

\section{Tissue-specific melon gene expression}

Melon cDNA libraries generated in the present study, as well as melon phloem EST libraries described in Omid et al. [29], were neither normalized nor subtracted; thus for these libraries, EST copy numbers can be used as an approximate estimation of gene expression levels in the corresponding tissues. The non-normalized and nonsubtracted melon cDNA libraries were prepared from the following seven tissues: leaf, flower, fruit, phloem, cotyledon, callus, and root. Statistical analysis identified a total of 175 tissue-specific genes, among which 49, 39, $20,25,9,15$, and 18 were leaf, flower, fruit, phloem, cotyledon, callus, and root-specific, respectively (Additional file 5). Heatmap representation of expression profiles of these tissue-specific genes is shown in Figure 4.

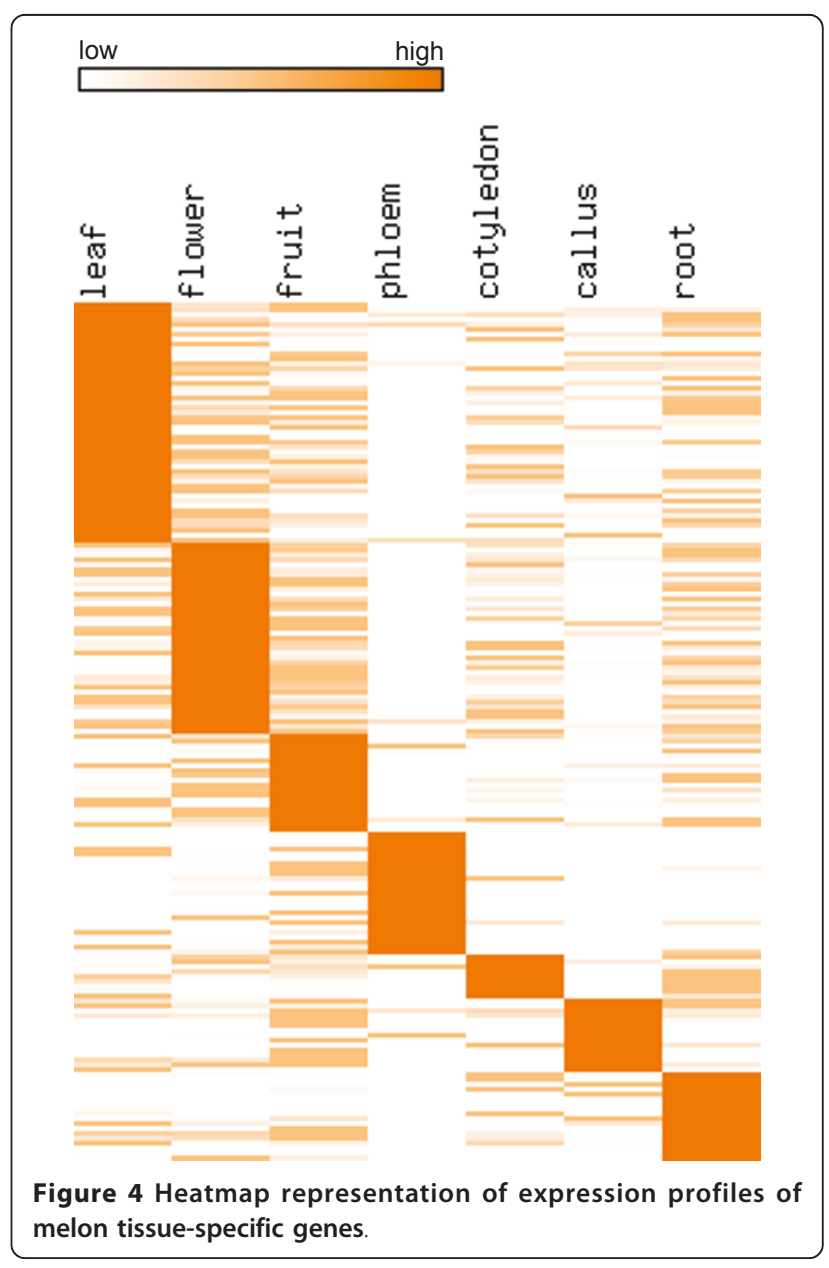


In most cases, genes expressed in specific tissues had putative functions or were involved in pathways known to be consistent with said tissue, e.g., leaf-specific genes were highly enriched with genes involved in photosynthesis, phloem-specific genes were highly enriched with genes encoding phloem filament proteins and phloem lectins, and callus-specific genes were highly enriched with genes involved in glycolysis, glucose metabolic process, hexose metabolic process, monosaccharide metabolic process, carbohydrate catabolic process, and alcohol metabolic process (Additional file 5). It is worth pointing out that some tissue-specific genes identified in leaf, cotyledon and root might be due to the infection of MNSV-M $\alpha$ 5. Indeed, functional analysis indicated that leaf, cotyledon and root-specific genes were enriched with GO terms such as response to stimulus and defense response (Additional file 5).

It is worth noting that one of the fruit-specific genes encoded 1-aminocyclopropane-1-carboxylate oxidase (ACO), the final enzyme in the biosynthesis of ethylene which is a plant hormone that regulates ripening of climacteric fruits [57]. Further detailed digital expression analysis of this gene (MU46283) revealed that, as expected, the gene was predominantly expressed in fruits of melon cultivars Dulce and Vedrantais, both of which are climacteric fruits; while none or very few ACO transcripts were detected in fruits of the two nonclimacteric cultivars, PI161375 and Piel de Sapo T-111. In addition, two genes (MU45060 and MU46015) encoding acyl carrier proteins (ACPs) were highly and exclusively expressed in fruit tissues. ACPs are essential components of the fatty acid synthase complex and may be required to maintain the production of fruit aroma volatiles [58].

Interestingly, we found that genes involved in nucleosome and chromatin assembly (e.g., histones) and translation process (e.g., ribosomal proteins) were highly enriched in the list of flower-specific genes (Additional file 5). However, the exact role of these flower-specific genes in melon flower development remains unclear and further studies are required to clarify their functions in flower development.

\section{Marker discovery from melon EST sequences}

Molecular markers are valuable resources for constructing high-density genetic maps, facilitating crop breeding and identifying traits of interest. Early melon genetic maps mainly used markers of Restriction Fragment Length Polymorphism (RFLP), Amplified Fragment Length Polymorphism (AFLP), and Random Amplified Polymorphic DNA (RAPD). However these types of markers are not user friendly as they are either labor intensive to generate, harbor low rates of polymorphism in melon [59], or are not readily transferred to other genotypes and populations [60]. With the accumulation of sequence information in melon during the past several years, markers of simple sequence repeats (SSRs) and single nucleotide polymorphisms (SNPs) are becoming more widely used in construction of melon genetic maps. These markers have the following advantages: they are hypervariable, multiallelic, codominant, locus-specific, and evenly distributed throughout the genome [60], and for markers derived from ESTs, they are directly linked to expressed genes. The melon EST sequence information generated in this and other studies has served as a major resource to generate new molecular markers (mainly SSRs and SNPs). Several recently constructed melon high-density genetic maps have already utilized SSR and SNP markers derived from EST sequences generated in the present study $[18,19]$.

We first screened melon unigenes for the presence of di-, tri-, tetra-, penta- and hexa-nucleotide SSR motifs. We retrieved 4,068 SSR motifs in 3,279 melon unigenes. The major types of melon SSR motifs were tri-nucleotide, followed by di-nucleotide, tetra-nucleotide, pentanucleotide and hexa-nucleotide (Table 4). The most frequent SSR motif was AAG/CTT $(1,269 ; 31.2 \%)$, followed by AG/CT $(1,134 ; 27.9 \%)$, AT/AT $(364 ; 8.9 \%)$ and AAT/ ATT (120; 2.9\%). CG/CG (3) was the least frequent SSR motif identified in melon unigenes; possibly due to the fact that CpG sequences are normally highly methylated, which may further inhibit transcription [61]. These statistics are in agreement with previous reports of other plant species $[26,62]$. Primer pairs were designed for SSR motifs that had sufficient flanking sequences. The complete list of SSR motifs and their corresponding primer pair information is provided in Additional file 6 .

ESTs generated in this (Table 1) and other studies $[24,28,29]$ were from a diversity of melon cultivars. We expected that SNPs would be enriched in the melon EST dataset. Using very stringent criteria (see Methods for details), we identified a total of 3,073 high-quality SNPs in 1,331 unigenes, among which 1,972 were transitions, 976 were transversions, and 125 were single-base insertions or deletions (Table 5). The most frequent SNPs were $C$ to $T$ transitions $(1,108 ; 36.1 \%)$, followed by $A$ to $G$ transitions $(864 ; 28.1 \%$ ) (Table 5 ). The complete list of SNPs identified from melon ESTs is

Table 4 Statistics of melon simple sequence repeats (SSRs)

\begin{tabular}{ll}
\hline Unit size & Number of SSRs \\
\hline di-nucleotide & 1657 \\
tri-nucleotide & 2157 \\
tetra-nucleotide & 124 \\
penta-nucleotide & 51 \\
hexa-nucleotide & 79 \\
\hline
\end{tabular}


Table 5 Statistics of melon single nucleotide polymorphisms (SNPs)

\begin{tabular}{llll}
\hline SNP & No. SNPs & type & total \\
\hline$A->G$ & 864 & Transition & 1972 \\
$C \rightarrow T$ & 1108 & & \\
\hline$A->C$ & 255 & & \\
$A->T$ & 289 & transversion & 976 \\
$C \rightarrow G$ & 210 & & \\
$G-T$ & 222 & & \\
\hline$T->-$ & 40 & & 125 \\
$A->-$ & 38 & indel & \\
$G->-$ & 23 & & \\
$C->-$ & 24 & & \\
\hline
\end{tabular}

provided in Additional file 7. Detailed information including alignments of sequences containing each individual SNP is also available at the Cucurbit Genomics Database [28]. Both SSRs and SNPs identified in the present study represent an important resource for genetic linkage mapping and marker-assisted breeding in melon and closely related crops. As stated above, they have already been used for these purposes.

\section{Conclusion}

We present the analysis of more than 71,000 and 22,000 melon ESTs from eleven full-length enriched and four standard cDNA libraries, respectively. These libraries were constructed from a range of tissues and melon genotypes. Analysis of approximately 1,400 melon fulllength transcripts identified from this EST collection indicated that melon transcripts had 5' and 3' UTRs of similar size as those of tomato, while longer than those of other dicot plants that we investigated. Comparative analysis between melon ESTs and other plant genomes allowed us to identify a number of highly conserved gene families across the plant kingdom, as well as gene families specific to fleshy-fruit bearing plants, to the Cucurbitaceae family, and to melon. Digital expression analysis identified genes showing significant tissue-specific expression and this resource remains to be further exploited from the perspective of mining expression data. Furthermore, SSR and SNP markers were also identified in this melon EST collection and recent research activities have begun to utilize these resources to construct high-density genetic maps $[18,19]$. Overall the availability of a large collection of melon ESTs from full-length enriched and standard cDNA libraries will not only facilitate the annotation of the melon genome, which is currently being sequenced by the Spanish Genomics Initiative, but also provide a valuable resource for further functional and comparative genomics analysis, and for future improvement of breeding programs of melon and closely related species.

\section{Methods}

\section{Plant material}

Fruits of the four genotypes were collected at four developmental stages: 10, 20, 30 Days After Anthesis (DAA) and at the mature stage. The mature stage was determined based on the formation of the abscission zone in the two climacteric genotypes Dulce and Vedrantais (42 and 32 DAA, respectively) and based on highest Total Soluble Solids (TSS) for the two non-climacteric fruits PI161375 and Piel de sapo (42 and 45 DAA, respectively). Hermaphrodite flowers were collected on secondary axes at three developmental stages, C1, C3, and C5, which correspond to initial, medium and late developmental stages of flowers before anthesis, respectively (Caño-Delgado, unpublished). Specifically, $\mathrm{C} 1$ is the most initial stage where the flowers are around $1 \mathrm{~mm}$ in the longitudinal axis, $\mathrm{C} 3$ is the stage where the future fruit shape is already defined and first stamens are visible, and C5 is the stage just before anthesis (1-2 cm). MNSV-M $\alpha 5$ infected cotyledons, leaves and roots were produced from melon cultivar Piel de Sapo T111 grown in growth chamber with a 16 -hour, $25^{\circ} \mathrm{C}$ light and 8 -hour, $18^{\circ} \mathrm{C}$ dark regime. Specifically, nine-day old cotyledons were inoculated mechanically with fresh inoculums of MNSV-M $\alpha 5$ and harvested after 4 days when necrotic lesions started to appear with high incidence. Leaves and roots were harvest 10 and 8-10 days after inoculation with MNSVM 5 , respectively. Undifferentiated callus growth was induced from cotyledon sections of the four cultivars (Dulce, Piel de Sapo T111, PI161375, and Vedrantais). Fifty seeds from each genotype were surfaced-sterilized in $70 \%$ ethanol for $2 \mathrm{~min}$, followed by $1 \%(\mathrm{w} / \mathrm{v}) \mathrm{NaOCl}$ with $0.1 \%(\mathrm{v} / \mathrm{v})$ Tween-20 for $20 \mathrm{~min}$, and rinsed three times with sterile distilled water. Under a dissecting microscope, seed coats were removed, a small incision was done on the integuments, and embryos were hydrated overnight in sterile distilled water. Embryo axis was removed from the de-coated seeds. Depending on the genotype, four to six transversal cotyledon sections were dissected from each seed and cultured in Petri dishes containing callus induction medium. Cultures were incubated in the dark, at $28^{\circ} \mathrm{C}$, and subcultured every three weeks to fresh medium. Callus induction medium was the MS (Murashige and Skoog), supplemented with $30 \mathrm{~g} \cdot \mathrm{L}^{-1}$ sucrose, $8 \mathrm{~g} \cdot \mathrm{L}^{-1}$ Bacto agar (Difco Laboratories, Detroit), 5uM 2,4-dichlorophenoxyacetic acid (2,4-D), and $1 \mathrm{uM}$ Kinetin (6-furfurylaminopurine). Five months after initiation, 100 Petri dishes, 10 -cm-wide, with six to eight calli were produced from each genotype.

\section{Total RNA preparation, CDNA library construction and cDNA clone sequencing}

Total RNAs from callus and MNSV-infected tissues were extracted following the TRI-reagent (SIGMA) 
protocol, including two additional chloroform purification steps. Fruit total RNAs were prepared from slices of the fruit that included both flesh and rind using the protocol described by Portnoy et al. [25]. Melon flower total RNA was extracted from hermaphrodite flowers using TRIzol reagent (Invitrogen) and chlorophorm, following the protocol described by Cuperus et al. [63].

All RNA samples were submitted to one extra cleaning step on RNeasy columns (Qiagen) and purified on a poly(A) track system (Promega). For cDNA library construction, fruit and flower RNAs were pooled, respectively, by mixing equal amount of RNA from each developmental stage. Full-length enriched cDNA libraries were constructed with the RNA Captor protocol, as described previously [64], and the four standard callus cDNA libraries were constructed using the pBluescript II XR cDNA Library Construction Kit (Stratagene) according to the manufacturer's instructions. A subset of clones was randomly selected from each cDNA library. Clones from full-length enriched cDNA libraries were sequenced at Genoscope (Evry, France) and those from standard cDNA libraries at Arizona Genome Institute.

\section{EST sequence processing, assembly, and annotation}

The raw chromatogram files were base-called with phred [65]. Vector, adaptor and low-quality bases (a 20bp window with an average error rate $>0.01$ ) were trimmed from the raw EST sequences using LUCY [66]. The resulting sequences were then screened against the NCBI UniVec database, E. coli genome, and melon ribosomal RNA sequences using SeqClean [67], to remove possible contaminations of these sequences. Sequences shorter than $100 \mathrm{bp}$ were discarded. The resulting high quality melon ESTs have been deposited in GenBank dbEST database under accession numbers JG463773JG557528 and are also available at the Cucurbit Genomics Database [28].

Melon ESTs were assembled into unigenes using iAssembler [68] with minimum overlap of $40 \mathrm{bp}$ and minimum percent identity of 97 . Melon unigene sequences were compared against GenBank non-redundant (nr) and UniProt [69] protein databases using the NCBI BLAST program with a cutoff e value of $1 \mathrm{e}-5$. The unigene sequences were translated into proteins using ESTScan [52] and the translated proteins were then compared to pfam domain database [30] using HMMER3 [70]. Gene Ontology (GO) terms and plantspecific GO slim ontology [31] were assigned to each unigene based on terms annotated to its corresponding homologues in the UniProt database and domains in pfam database. Melon biochemical pathways were predicted from the unigenes using the Pathway Tools program [32] and a melon biochemical pathway database was constructed and is available at the Cucurbit Genomics Database [28].

\section{Full-length transcript identification and analysis}

Unigenes containing both 5' and 3' sequences of at least one clone from the full-length enriched cDNA libraries were identified as full-length transcripts. The complete CDS were identified using the getorf application in the EMBOSS package [71]. CDS were also identified based on the ESTScan translations and CDS identified from the two approaches were integrated. 5' and 3' UTRs were then extracted from each candidate full-length transcript. Codon usages were calculated with the cusp program in the EMBOSS package [71].

\section{Comparative genomics analysis}

Melon unigenes were compared to protein databases of fourteen plant species whose genomes have been fully sequenced (Additional file 2) using the NCBI BLAST program with an e value cutoff of 1e-5. Furthermore, ortholog groups of protein sequences for melon (ESTScan translated proteins), Arabidopsis, rice, cucumber, and grape were identified using the orthoMCL program, which performs an all-against-all BLAST comparison of protein sequences with subsequent Tribe-Markov clustering [51. Venn diagram showing the distribution of shared gene families among melon, Arabidopsis, rice, cucumber and grape was created with Venn Diagrams [72]. Enriched GO terms of melon unigenes in each list of specific ortholog groups were identified using GO:: TermFinder [73] with corrected p values (False Discovery Rate (FDR); [74]) less than 0.05 .

\section{Identification of tissue-specific genes}

All normalized or subtracted cDNA libraries (e.g., libraries described in Gonzalez-Ibeas et al [24]) were excluded in the digital expression analysis. Pair-wise comparisons between fruit, flower, callus, leaf, root, cotyledon (Table 1), and phloem [29] were performed with the R statistic described in Stekel et al. [75] to identify differentially expressed genes. Only genes with a total of at least five EST members in the two compared tissues were included in the analysis. Raw p values from the $\mathrm{R}$ statistic were corrected for multiple testing using the FDR [74]. Tissue-specific genes were identified if the genes were significantly up-regulated (ratio $>2$ and FDR $<0.05$ ) in the tissue when compared to all other tissues. Enriched GO terms in each list of tissue-specific genes were identified using GO:: TermFinder [73], requiring $\mathrm{p}$ values adjusted for multiple testing (FDR) to be less than 0.05 .

\section{Identification of SSRs and SNPs}

SSRs in melon unigene sequences were identified using the MISA program [76]. The minimum repeat number 
was six for dinucleotide and five for tri-, tetra-, pentaand hexa-nucleotide. Primer pairs flanking each SSR loci were designed using the Primer3 program [77].

SNPs in the cDNA sequences between different melon cultivars were identified with PolyBayes [78], which takes into account both the depth of the coverage and quality of the bases. To further eliminate errors introduced by PCR amplification during the cDNA synthesis step and to distinguish true SNPs from allele differences, we filtered PolyBayes results and only kept SNPs meeting both of the following two criteria: 1 ) at least $2 \mathrm{X}$ coverage at the potential SNP site for each cultivar; 2) no same bases at the potential SNP site between the two compared cultivars. The detailed information of all melon SSRs and SNPs is freely available at the Cucurbit Genomics Database [28].

\section{Additional material}

Additional file 1: Codon usages of melon and Arabidopsis coding sequences. The table provides the statistics of codon usages of melon and Arabidopsis coding sequences.

Additional file 2: Plant protein databases used for comparative genomics analysis. The table provides the list of protein databases of plants with fully sequenced genomes that were used in the comparative analysis of melon unigenes.

Additional file 3: Comparative analysis of melon unigenes. The table provides the statistics of comparison between melon unigenes and fourteen plant protein databases using the BLAST program.

Additional file 4: Enriched Gene Ontology (GO) terms in gene families. The table provides the list of enriched GO terms identified from melon unigenes in gene families specific to the Cucurbitaceae family, to the fleshy fruit-bearing plant species, and common across the five plant species, respectively.

Additional file 5: Tissue-specific genes. The file provides the list of genes that have tissue-specific expression and the list of $\mathrm{GO}$ terms enriched in each list of tissue-specific genes.

Additional file 6: Melon SSRs. The table provides the list of SSRs identified from melon ESTs, their motif sequences and surrounding primer pair information.

Additional file 7: melon SNPs. The table provides the list of SNPS identified from melon ESTs.

\section{Acknowledgements \\ This work was supported by Research Grant Award No. IS-4223-09C from BARD, the United States-Israel Binational Agricultural Research and Development Fund, and by SNC Laboratoire ASL, de Ruiter Seeds B.V., Enza Zaden B.V., Gautier Semences S.A., Nunhems B.V., Rijk Zwaan B.V., Sakata Seed Inc, Semillas Fitó S.A., Seminis Vegetable Seeds Inc, Syngenta Seeds B. V., Takii and Company Ltd, Vilmorin and Cie S.A. and Zeraim Gedera Ltd (all of them as part of the support to ICUGI). CC was supported by CNRS ERL 8196.}

\section{Author details}

'URGV Plant Genomics, Unité de Recherche en Génomique Végétale, UMR1165 ERL8196 INRA-UEVE-CNRS. 2, Rue Gaston Crémieux, 91057 Evry, France. ${ }^{2}$ Molecular and Cellular Imaging Center, The Ohio State University, OARDC, 1680 Madison Ave, Wooster, OH 44691, USA. ${ }^{3}$ Boyce Thompson Institute, Cornell University, Ithaca, NY 14853, USA. ${ }^{4}$ Centro de Edafología y Biología Aplicada del Segura (CEBAS), Consejo Superior de Investigaciones
Científicas (CSIC), Apdo. Correos 164, 30100 Espinardo, Murcia, Spain. ${ }^{5}$ IRTA Center for Research in Agricultural Genomics CSIC-IRTA-UAB, Campus UAB, Edifici CRAG, 08193 Bellaterra (Barcelona), Spain. ${ }^{6}$ Department of Vegetable Research, Agricultural Research Organization, Newe Ya'ar Research Center, P. O. Box 1021, Ramat Yishay, 30095, Israel. 'Department de Genètica Molecular, Center for Research in Agricultural Genomics CSIC-IRTA-UAB, Campus UAB, Edifici CRAG, 08193 Bellaterra (Barcelona), Spain. ${ }^{8}$ Department of Plant Production, College of Food and Agricultural Sciences, King Saud University, Riyadh Saudi Arabia. ${ }^{9}$ USDA Robert W. Holley Center for Agriculture and Health, Tower Road, Ithaca, NY 14853, USA. ${ }^{10}$ Seminis Vegetable Seeds, 37437 State Highway 16 Woodland, CA 95695, USA.

\section{Authors' contributions}

ZF, CC, TJ, JGM, ABe, JJG and MAA conceived and designed the study. JGM coordinated the ICUGI project. VT, RDS, VP, AMC, AICD and NK collected tissues and prepared RNA samples. CC, TJ, DJ, ABo and MEH constructed CDNA libraries. YZ, MH and ZF performed data analysis. ZF and CC wrote the manuscript. All authors approved the final manuscript.

Received: 22 March 2011 Accepted: 20 May 2011

Published: 20 May 2011

\section{References}

1. Jeffrey C: A new system of Cucurbitaceae. Bot Zhurn 2005, 90:332-335.

2. Jeffrey C, De Wilde WJJO: A review of the subtribe Thladianthinae (Cucurbitaceae). Bot Zhurn 2006, 91:766-776.

3. Boualem A, Fergany M, Fernandez R, Troadec C, Martin A, et al: A conserved mutation in an ethylene biosynthesis enzyme leads to andromonoecy in melons. Science 2008, 321:836-838.

4. Martin A, Troadec C, Boualem A, Rajab M, Fernandez R, et al: A transposoninduced epigenetic change leads to sex determination in melon. Nature 2009, 461:1135-1138.

5. Haritatos E, Keller F, Turgeon R: Raffinose oligosaccharide concentrations measured in individual cell and tissue types in Cucumis melo L. Leaves: implication for phloem loading. Planta 1996, 198:614-622.

6. Gomez G, Torres H, Pallas V: Identification of translocatable RNA-binding phloem proteins from melon, potential components of the longdistance RNA transport system. Plant J 2005, 41:107-116.

7. Nunez-Palenius HG, Gomez-Lim M, Ochoa-Alejo N, Grumet R, Lester G, Cantliffe DJ: Melon fruits: genetic diversity, physiology, and biotechnology features. Crit Rev Biotechnol 2008, 28:13-55.

8. Giovannoni JJ: Fruit ripening mutants yield insights into ripening control. Curr Opin Plant Biol 2007, 10:283-289.

9. Gonda I, Bar E, Portnoy V, Lev S, Burger J, Schaffer AA, Tadmor Y Gepstein S, Giovannoni JJ, Katzir N, Lewinsohn E: Branched-chain and aromatic amino acid catabolism into aroma volatiles in Cucumis melo L. fruit. J Exp Bot 2010, 61:1111-1123.

10. Dai N, Cohen S, Portnoy V, Tzuri G, Harel-Beja R, Pompan-Lotan M, Carmi N, Zhang G, Diber A, Pollock S, et al: Metabolism of soluble sugars in developing melon fruit: a global transcriptional view of the metabolic transition to sucrose accumulation. Plant Mol Biol 2011.

11. Arumuganathan K, Earle ED: Nuclear DNA content of some important plant species. Plant Molecular Biology Reporter 1991, 9:208-218.

12. van Leeuwen $H$, Monfort $A$, Zhang $H B$, Puigdomenech $P$ : Identification and characterization of a melon genomic region containing a resistance gene cluster from a constructed BAC library. Microcolinearity between Cucumis melo and Arabidopsis thaliana. Plant Mol Biol 2003, 51:703-718.

13. Morales M, Orjeda G, Nieto C, van Leeuwen $H$, Monfort A, et al: A physical map covering the nsv locus that confers resistance to Melon necrotic spot virus in melon (Cucumis melo L.). Theor Appl Genet 2005, 111:914-922.

14. Gonzalez VM, Rodriguez-Moreno L, Centeno E, Benjak A, Garcia-Mas J, Puigdomenech P, Aranda MA: Genome-wide BAC-end sequencing of Cucumis melo using two BAC libraries. BMC Genomics 2011, 11:11.

15. Gonzalez VM, Garcia-Mas J, Arus P, Puigdomenech P: Generation of a BAC-based physical map of the melon genome. BMC Genomics 2010, 11:339.

16. Perin C, Gomez-Jimenez M, Hagen L, Dogimont C, Pech JC, et al: Molecular and genetic characterization of a non-climacteric phenotype in melon reveals two loci conferring altered ethylene response in fruit. Plant Physiol 2002, 129:300-309. 
17. Fernandez-Silva I, Eduardo I, Blanca J, Esteras C, Pico B, et al: Bin mapping of genomic and EST-derived SSRs in melon (Cucumis melo L.). Theor Appl Genet 2008, 118:139-150.

18. Deleu W, Esteras C, Roig C, Gonzalez-To M, Fernandez-Silva I, GonzalezIbeas D, Blanca J, Aranda MA, Arus P, Nuez F, Monforte AJ, Pico MB, GarciaMas J: A set of EST-SNPs for map saturation and cultivar identification in melon. BMC Plant Biol 2009, 9:90.

19. Harel-Beja R, Tzuri G, Portnoy V, Lotan-Pompan M, Lev S, Cohen S, Dai N, Yeselson L, Meir A, Libhaber SE, et al: A genetic map of melon highly enriched with fruit quality QTLs and EST markers, including sugar and carotenoid metabolism genes. Theor Appl Genet 2010, 121:511-533.

20. Mascarell-Creus A, Canizares J, Vilarrasa-Blasi J, Mora-Garcia S, Blanca J, et al: An oligo-based microarray offers novel transcriptomic approaches for the analysis of pathogen resistance and fruit quality traits in melon (Cucumis melo L.). BMC Genomics 2009, 10:467.

21. Dahmani-Mardas F, Troadec C, Boualem A, Lévêque S, Alsadon AA, et al: Engineering melon plants with improved fruit shelf life using the TILLING Approach. PLOS ONE 2010, 5:e15776.

22. Huang S, Li R, Zhang Z, Li L, Gu X, Fan W, Lucas WJ, Wang X, Xie B, Ni P, et al: The genome of the cucumber, Cucumis sativus L. Nat Genet 2009, 41:1275-1281.

23. Rudd S: Expressed sequence tags: alternative or complement to whole genome sequences? Trends Plant Sci 2003, 8:321-329.

24. Gonzalez-Ibeas D, Blanca J, Roig C, Gonzalez-To M, Pico B, Truniger V, Gomez P, Deleu W, Cano-Delgado A, Arus P, et al: MELOGEN: an EST database for melon functional genomics. BMC Genomics 2007, 8:306.

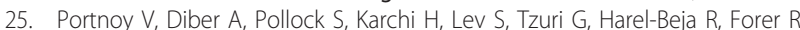
Portnoy VH, Lewinsohn E, Tadmor Y, Burger J, Schaffer A, Katzir N: Use of non-normalized, non-amplified cDNA for 454-based RNA-seq of fleshy melon fruit. The Plant Genome 2011.

26. Guo S, Zheng Y, Joung JG, Liu S, Zhang Z, Crasta OR, Sobral BW, Xu Y, Huang S, Fei Z: Transcriptome sequencing and comparative analysis of cucumber flowers with different sex types. BMC Genomics 2010, 11:384.

27. Blanca J, Cañizares J, Roig C, Ziarsolo P, Nuez F, Picó B: Transcriptome characterization and high throughput SSRs and SNPs discovery in Cucurbita pepo (Cucurbitaceae). BMC Genomics 2011, 12:104.

28. Cucurbit Genomics Database. [http://www.icugi.org]

29. Omid A, Keilin T, Glass A, Leshkowitz D, Wolf S: Characterization of phloem-sap transcription profile in melon plants. J Exp Bot 2007, 58:3645-3656

30. Finn RD, Mistry J, Tate J, Coggill P, Heger A, Pollington JE, Gavin OL, Gunasekaran P, Ceric G, Forslund K: The Pfam protein families database. Nucleic Acids Res 2010, 38:D211-222.

31. Plant specific GO slims. [http://www.geneontology.org/GO.slims.shtml].

32. Karp PD, Paley S, Romero P: The Pathway Tools software. Bioinformatics 2002, 18:S225-S232.

33. Aoki K, Yano K, Suzuki A, Kawamura S, Sakurai N, Suda K, Kurabayashi A, Suzuki T, Tsugane T, Watanabe M, et al: Large-scale analysis of full-length cDNAs from the tomato (Solanum lycopersicum) cultivar Micro-Tom, a reference system for the Solanaceae genomics. BMC Genomics 2010, 11:210.

34. Yamada K, Lim J, Dale JM, Chen H, Shinn P, Palm CJ, Southwick AM Wu HC, Kim C, Nguyen M, et al: Empirical analysis of transcriptional activity in the Arabidopsis genome. Science 2003, 302:842-846.

35. Umezawa T, Sakurai T, Totoki Y, Toyoda A, Seki M, Ishiwata A, Akiyama K, Kurotani A, Yoshida T, Mochida K, et al: Sequencing and analysis of approximately 40,000 soybean cDNA clones from a full-length-enriched cDNA library. DNA Res 2008, 15:333-346.

36. Ralph SG, Chun HJ, Cooper D, Kirkpatrick R, Kolosova N, Gunter L, Tuskan GA, Douglas CJ, Holt RA, Jones SJ, et al: Analysis of 4,664 highquality sequence-finished poplar full-length CDNA clones and their utility for the discovery of genes responding to insect feeding. BMC Genomics 2008, 9:57.

37. Jia J, Fu J, Zheng J, Zhou X, Huai J, Wang J, Wang M, Zhang Y, Chen X, Zhang J, Zhao J, Su Z, LV Y, Wang G: Annotation and expression profile analysis of 2073 full-length cDNAs from stress-induced maize (Zea mays L.) seedlings. Plant J 2006, 48:710-727.

38. Arabidopsis Genome Initiative: Analysis of the genome sequence of the flowering plant Arabidopsis thaliana. Nature 2000, 408:796-815.

39. International Rice Genome Sequencing Project: The map-based sequence of the rice genome. Nature 2005, 436:793-800.
40. Tuskan GA, Difazio S, Jansson S, Bohlmann J, Grigoriev I, Hellsten U, Putnam N, Ralph S, Rombauts S, Salamov A, et al: The genome of black cottonwood, Populus trichocarpa (Torr. \& Gray). Science 2006, 313:1596-1604.

41. Jaillon O, Aury JM, Noel B, Policriti A, Clepet C, Casagrande A, Choisne N, Aubourg S, Vitulo N, Jubin C, et al: The grapevine genome sequence suggests ancestral hexaploidization in major angiosperm phyla. Nature 2007, 449:463-467.

42. Ming $R$, Hou S, Feng $Y$, Yu Q, Dionne-Laporte A, Saw JH, Senin P, Wang W, Ly BV, Lewis $K L$, et al: The draft genome of the transgenic tropical fruit tree papaya (Carica papaya Linnaeus). Nature 2008, 452:991-996.

43. Paterson AH, Bowers JE, Bruggmann R, Dubchak I, Grimwood J, Gundlach H, Haberer G, Hellsten U, Mitros T, Poliakov A, et al: The Sorghum bicolor genome and the diversification of grasses. Nature 2009, 457:551-556.

44. Schnable PS, Ware D, Fulton RS, Stein JC, Wei F, Pasternak S, Liang C, Zhang J, Fulton L, Graves TA, et al: The B73 maize genome: complexity, diversity, and dynamics. Science 2009, 326:1112-1115.

45. Schmutz J, Cannon SB, Schlueter J, Ma J, Mitros T, Nelson W, Hyten DL, Song Q, Thelen JJ, Cheng J, et al: Genome sequence of the palaeopolyploid soybean. Nature 2010, 463:178-183.

46. International Brachypodium Initiative: Genome sequencing and analysis of the model grass Brachypodium distachyon. Nature 2010, 463:763-768.

47. Velasco R, Zharkikh A, Affourtit J, Dhingra A, Cestaro A, Kalyanaraman A, Fontana P, Bhatnagar SK, Troggio M, Pruss D, et al: The genome of the domesticated apple (Malus x domestica Borkh.). Nat Genet 2010, 42:833-839.

48. Chan AP, Crabtree J, Zhao Q, Lorenzi H, Orvis J, Puiu D, Melake-Berhan A, Jones KM, Redman J, Chen G, et al: Draft genome sequence of the oilseed species Ricinus communis. Nat Biotechnol 2010, 28:951-956.

49. Shulaev V, Sargent DJ, Crowhurst RN, Mockler TC, Folkerts O, Delcher AL, Jaiswal P, Mockaitis K, Liston A, Mane SP, et al: The genome of woodland strawberry (Fragaria vesca). Nat Genet 2011, 43:109-116.

50. Argout X, Salse J, Aury JM, Guiltinan MJ, Droc G, Gouzy J, Allegre M, Chaparro C, Legavre T, Maximova SN, et al: The genome of Theobroma cacao. Nat Genet 2011, 43:101-108.

51. Li L, Stoeckert CJJ, Roos DS: OrthoMCL: identification of ortholog groups for eukaryotic genomes. Genome Res 2003, 13:2178-2189.

52. Iseli C, Jongeneel CV, Bucher P: ESTScan: a program for detecting, evaluating, and reconstructing potential coding regions in EST sequences. Proc Int Conf Intell Syst Mol Biol 1999, 138-148.

53. Giovannoni JJ: Genetic regulation of fruit development and ripening. Plant Cell 2004, 16:S170-S180.

54. Tanurdzic M, Banks JA: Sex-determining mechanisms in land plants. Plant Cell 2004, 16:S61-S71.

55. Heslop-Harrison J: The experimental modification of sex expression in flowering plants. Biol Rev 1957, 32:38-90.

56. Korpelainen H: Labile sex expression in plants. Biol Rev 1998, 73:157-180.

57. Yang SF, Hoffman NE: Ethylene biosynthesis and its regulation in higherplants. Annu Rev Plant Physiol Plant Mol Biol 1984, 35:155-189.

58. Schwab W, Davidovich-Rikanati R, Lewinsohn E: Biosynthesis of plantderived flavor compounds. The Plant Journal 2008, 54:712-732.

59. Shattuck-Eidens DM, Bell RN, Neuhausen SL, Helentjaris T: DNA sequence variation within maize and melon: observations from polymerase chain reaction amplification and direct sequencing. Genetics 1990, 126:207-217.

60. Ezura $\mathrm{H}$, Fukino $\mathrm{N}$ : Research tools for functional genomics in melon (Cucumis melo L.): Current status and prospects. Plant Biotechnology 2009, 26:359-368.

61. Lister R, O'Malley RC, Tonti-Filippini J, Gregory BD, Berry CC, Millar AH, Ecker JR: Highly integrated single-base resolution maps of the epigenome in Arabidopsis. Cell 2008, 133:523-536.

62. Kantety RV, La Rota M, Matthews DE, Sorrells ME: Data mining for simple sequence repeats in expressed sequence tags from barley, maize, rice, sorghum and wheat. Plant Mol Biol 2002, 48:501-510.

63. Cuperus JT, Montgomery TA, Fahlgren N, Burke RT, Townsend T, Sullivan CM, Carrington JC: Identification of MIR390a precursor processing-defective mutants in Arabidopsis by direct genome sequencing. Proc Natl Acad Sci USA 2010, 107:466-471.

64. Clepet C: RNA Captor, a tool for RNA characterization. PLOS ONE 2011, 6 : e18445.

65. Ewing $B$, Hillier L, Wendl MC, Green P: Base-calling of automated sequencer traces using phred. I. Accuracy assessment. Genome Res 1998, 8:175-185 
66. Chou HH, Holmes MH: DNA sequence quality trimming and vector removal. Bioinformatics 2001, 17:1093-1104.

67. SeqClean program. [http://compbio.dfci.harvard.edu/tgi/software].

68. iAssembler program. [http://bioinfo.bti.cornell.edu/tool/iAssembler].

69. Apweiler R, Martin MJ, O'Donovan C, Magrane M, Alam-Faruque $Y$, Antunes R, Barrell D, Bely B, Bingley M, Binns D, et al: The Universal Protein Resource (UniProt) in 2010. Nucleic Acids Res 2010, 38:D142-148.

70. HMMER3. [http://hmmer.janelia.org].

71. Rice P, Longden I, Bleasby A: EMBOSS: The European Molecular Biology Open Software Suite. Trends in Genetics 2000, 16:276-277.

72. Venn Diagrams. [http://bioinformatics.psb.ugent.be/webtools/Nenn]

73. Boyle El, Weng S, Gollub J, Jin H, Botstein D, Cherry JM, Sherlock G: GO:TermFinder: open source software for accessing Gene Ontology information and finding significantly enriched Gene Ontology terms associated with a list of genes. Bioinformatics 2004, 20:3710-3715.

74. Benjamini $Y$, Hochberg $Y$ : Controlling the false discovery rate: a practical and powerful approach to multiple testing. J R Stat Soc Ser B 1995, 57:289-300.

75. Stekel DJ, Git Y, Falciani F: The comparison of gene expression from multiple cDNA libraries. Genome Res 2000, 10:2055-2061.

76. MISA program. [http://pgrc.ipk-gatersleben.de/misa].

77. Primer3 program. [http://frodo.wi.mit.edu].

78. Marth GT, Korf I, Yandell MD, Yeh RT, Gu Z, Zakeri H, Stitziel NO, Hillier L, Kwok PY, Gish WR: A general approach to single-nucleotide polymorphism discovery. Nat Genet 1999, 23:452-456.

doi:10.1186/1471-2164-12-252

Cite this article as: Clepet et al:: Analysis of expressed sequence tags generated from full-length enriched CDNA libraries of melon. BMC Genomics 2011 12:252.

\section{Submit your next manuscript to BioMed Central} and take full advantage of:

- Convenient online submission

- Thorough peer review

- No space constraints or color figure charges

- Immediate publication on acceptance

- Inclusion in PubMed, CAS, Scopus and Google Scholar

- Research which is freely available for redistribution

Submit your manuscript at www.biomedcentral.com/submit
C Biomed Central 